\title{
The surgical management of pituitary apoplexy with occluded internal carotid artery and hidden intracranial aneurysm: illustrative case
}

\author{
*Jian-Dong Zhu, MD, Sungel Xie, MD, Ling Xu, MD, Ming-Xiang Xie, MD, and Shun-Wu Xiao, MD \\ Department of Neurosurgery, Affiliated Hospital of Zunyi Medical University, Guizhou, China
}

BACKGROUND Approximately $0.6 \%$ to $12 \%$ of cases of pituitary adenoma are complicated by apoplexy, and nearly $6 \%$ of pituitary adenomas are comorbid aneurysms. Occlusion of the internal carotid artery (ICA) with hidden intracranial aneurysm due to compression by an apoplectic pituitary adenoma is extremely rare; thus, the surgical strategy is also unknown.

OBSERVATIONS The authors reported the case of a 48-year-old man with a large pituitary adenoma with coexisting ICA occlusion. After endoscopic transnasal surgery, repeated computed tomography angiography (CTA) demonstrated reperfusion of the left ICA but with a new-found aneurysm in the left posterior communicating artery; thus, interventional aneurysm embolization was performed. With stable recovery and improved neurological condition, the patient was discharged for rehabilitation training.

LESSONS For patients with pituitary apoplexy accompanied by a rapid decrease of neurological conditions, emergency decompression through endoscopic endonasal transsphenoidal resection can achieve satisfactory results. However, with occlusion of the ICA by enlarged pituitary adenoma or pituitary apoplexy, a hidden but rare intracranial aneurysm may be considered when patients are at high risk of such vascular disease as aneurysm, and gentle intraoperative manipulations are required. Performing CTA or digital subtraction angiography before and after surgery can effectively reduce the missed diagnosis of comorbidity and thus avoid life-threatening bleeding events from the accidental rupture of an aneurysm.

https://thejns.org/doi/abs/10.3171/CASE20115

KEYWORDS pituitary apoplexy; intracranial aneurysm; surgical strategy; occluded internal carotid artery

Pituitary apoplexy is a clinical syndrome characterized by sudden headache, visual impairment, and ophthalmoplegia due to the rapid enlargement of a pituitary adenoma resulting from bleeding or infarction. ${ }^{1}$ Internal carotid artery (ICA) compression by a pituitary adenoma is relatively rare and usually asymptomatic. Most symptomatic patients reported in the literature presented secondary to pituitary apoplexy. ${ }^{2}$ After extensive literature review, approximately $0.6 \%$ to $12 \%$ of patients with pituitary adenoma have cases complicated by apoplexy, ${ }^{3}$ and nearly $6 \%$ of pituitary adenomas are comorbid with aneurysms. ${ }^{4-7}$ However, the occlusion of ICA with hidden intracranial aneurysm due to compression by an apoplectic pituitary adenoma is extremely rare; thus, the surgical strategy is also unknown. Here, we report a case involving apoplexy of a large pituitary adenoma with coexisting ICA occlusion and a posterior communicating aneurysm.

\section{Illustrative Case}

A 48-year-old man presented to our hospital with headache and vomiting for 5 days and drooping eyelid and decreasing left eye vision for 1 day. The patient was awake when admitted, with a Glasgow Coma Scale (GCS) score of 15. Physical examination indicated left eyelid ptosis, out-and-downward strabismus of left eyeball, bilateral pupils in different diameters (left, $4 \mathrm{~mm}$; right, $3 \mathrm{~mm}$ ), impaired direct/indirect light reflex of the left eye, and blindness of left eye without light perception. Computed tomography (CT) scans

ABBREVIATIONS ACOA = anterior communicating artery; $\mathrm{CT}=$ computed tomography; $\mathrm{CTA}=\mathrm{CT}$ angiography; DSA = digital subtraction angiography; $\mathrm{EETS}=$ endoscopic endonasal transsphenoidal; GCS = Glasgow Coma Scale; ICA = internal carotid artery; $\mathrm{MRI}=$ magnetic resonance imaging; PCOA = posterior communicating artery.

INCLUDE WHEN CITING Published August 2, 2021; DOI: 10.3171/CASE20115.

SUBMITTED December 3, 2020. ACCEPTED June 18, 2021.

* J.D.Z. and S.X. contributed equally to this work.

(C) 2021 The authors, CC BY-NC-ND 4.0 (http://creativecommons.org/licenses/by-nc-nd/4.0/). 
obtained at the emergency department showed a tumor in the sellar region and possible pituitary apoplexy (Fig. 1). On day 2 of admission, the patient's condition suddenly worsened with a new onset of disturbance of consciousness (GCS 11), hemiplegia (grade II muscle strength of the right limbs), and hyperthermia (the highest temperature noted was $39.5^{\circ} \mathrm{C}$ ). Emergency CT indicated increased intratumoral bleeding and cerebral infarction in the left basal ganglia (Fig. 1). Emergency CT angiography (CTA) showed no filling of contrast medium in the left ICA. The blood supply of the left cerebrum was compensated by contralateral blood flow through the anterior communicating artery (ACOA) (Fig. 2). Emergency magnetic resonance imaging (MRI) showed apoplexy of a large pituitary adenoma and no development of the left cavernous segment of the ICA due to external compression (Fig. 3). Emergency surgery with an endoscopic endonasal transsphenoidal (EETS) approach was planned. Intraoperatively, the tumor had a tough texture and gray-red color, and the interior was soft and delicate with contained mechanized hematoma and a rich blood supply. With careful manipulation, the tumor was removed using gross total resection (Fig. 3). Given the expected levels of pituitary hormones in this patient and the pathological result after surgery, the diagnosis of nonfunctional cell adenoma was considered.

On day 3 after surgery, the patient recovered consciousness; his drooping left eye improved, but his left eye movement was still tricky and he was without visual sense. The muscle strength of the right upper and lower limbs was grade II and III, respectively. Repeat cranial CTA showed a $5.0 \times 5.5$-mm aneurysm in the posterior communicating artery (PCOA) of the left ICA (Fig. 2). We performed emergency interventional aneurysm embolization. The patient's neurological dysfunction gradually improved, and his physical condition was stable. He was discharged from the hospital with full 10 days of hospitalization. In the third month of follow-up after surgery, the patient showed normal muscle strength in all limbs and improved oculomotor nerve palsy, but only light perception existed in his left eye. MRI did not demonstrate recurrent signal of tumor (Fig. 3).

\section{Discussion \\ Observations}

The incidence of pituitary apoplexy is $0.6 \%$ to $12 \%$ in patients with pituitary adenoma. ${ }^{8}$ Moreover, symptoms of pituitary apoplexy are associated with external compression due to a sharp increase in the volume of pituitary tumors, which can manifest as sudden headaches with common nausea and vomiting. ${ }^{9,10}$ If compression involves the optic nerve or optic chiasma or even the oculomotor nerve and normal pituitary tissue, a significant decrease in visual fields and visual acuity or even ophthalmoplegia and endocrinological deterioration may occur in patients.,11,12 If the compression involves the ICA, disturbed consciousness and neurological deficits caused by cerebral hemisphere ischemic infarction may arise. ${ }^{13}$
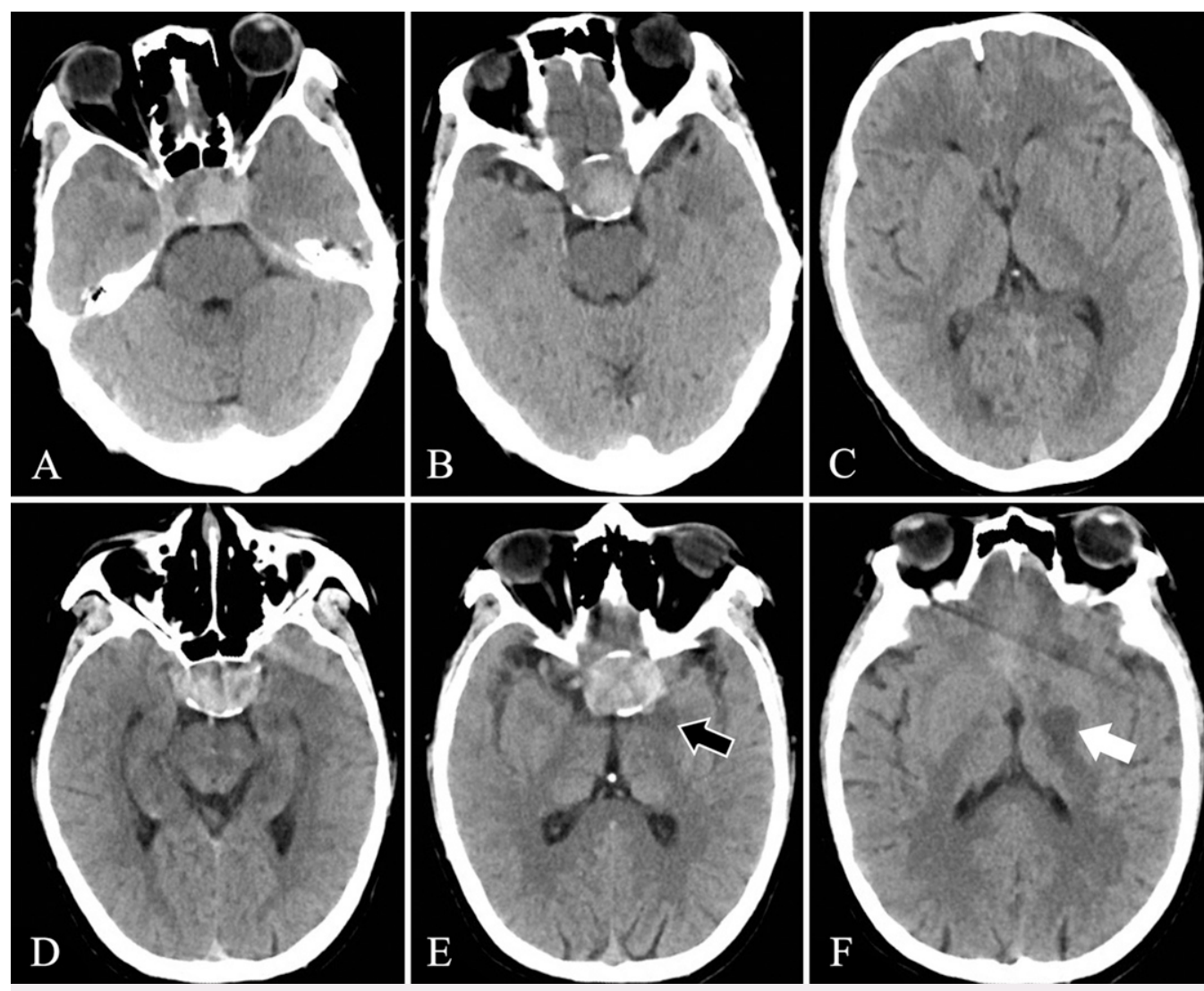

FIG. 1. Axial CT scans (A-C) on the day of admission showing a lesion in the pituitary fossa with a high-density bleeding signal. Axial CT images (D-F) on day 2 after admission illustrating that the size of the high-density bleeding signal in the lesion increased ( $E$, black arrow) and that an extra local low-density shadow came up in the left basal ganglia ( $\mathbf{F}$, white arrow). 

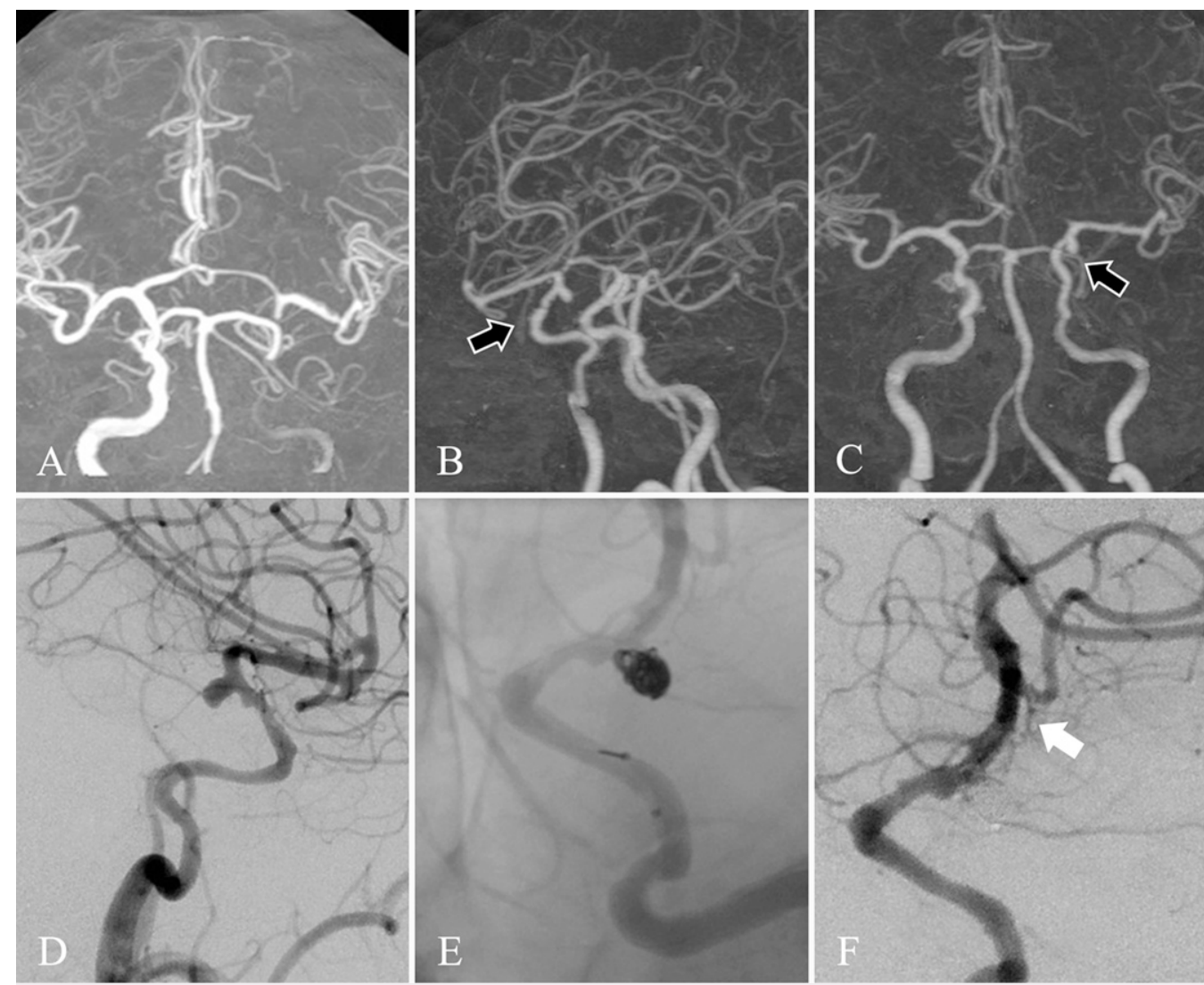

FIG. 2. Preoperative CTA (A) demonstrating the occluded left ICA with development of the middle cerebral artery due to the blood supply compensated by contralateral ICA circulation via ACOA. CTA (B and C) after resection of pituitary adenoma revealing the recanalization of the left ICA and an extra $5.0 \times 5.5-\mathrm{mm}$ aneurysm in the PCOA of the left ICA (black arrows). Endovascular images of interventional embolization of aneurysm (D-F) illustrating a saccate aneurysm $4.90 \times 5.28 \mathrm{~mm}$ in size and $4.05 \mathrm{~mm}$ in neck width at the original part of the anterior choroidal artery. Spring coils $(5 \times 150 \mathrm{~mm}$ and $2 \times 60 \mathrm{~mm})$ were smooth and filled with a dense embolism $(\mathbf{E})$. The aneurysm did not appear on the image after interventional embolization (F, white arrow).

Mainly, ICA occlusion caused by large pituitary adenoma is extremely rare, but with the occurrence of pituitary apoplexy, such occlusion may be at higher risk. ${ }^{2,13,14}$ In the present case, the patient initially reported the common symptoms of headache, visual loss, and oculomotor nerve palsy but without endocrinological changes. With the increase of bleeding in pituitary adenoma, cerebral infarction accompanied by hemiplegia and disorder of consciousness occurred due to the occlusion of the ICA. In addition, this patient experienced centric high fever when pituitary apoplexy deteriorated, which may be attributed to compression or ischemia of the hypothalamus.

Pituitary adenomas complicated by intracranial aneurysms have been reported, ranging from $2.3 \%$ to $8.3 \%$, and are the subject of increased attention. ${ }^{4-7}$ The comorbidity of pituitary adenoma and intracranial aneurysm is complex, and the priority of treatment is still controversial. Some experts believe that they can be treated simultaneously or that the aneurysms can be treated with endovascular intervention in the first stage and then the pituitary adenoma can be resected with transnasal endoscopic surgery, preventing lethal bleeding from a ruptured aneurysm. ${ }^{5}$ Other experts believe that no standard treatment exists for pituitary adenoma with an intracranial aneurysm and that personalized treatment should be administered according to size, location, and interrelationship of the tumors and patient condition. ${ }^{15}$ Nevertheless, for patients with pituitary apoplexy complicated by cerebral infarction due to occlusive ICA, there are still reports of new hemorrhagic transformation of the primary infarct after recanalization of the ICA. ${ }^{16}$ Thus, some researchers suggest that for patients with cerebral infarction caused by pituitary apoplexy combined with occlusive ICA, endoscopic surgery can be performed after a patient is stabilized to prevent cerebral hemisphere if there are no vision and visual field disorders hemorrhagic infarction from maintaining a stable cerebral circulation. ${ }^{13}$ Depending on these views above, in our opinion, treatment of the coexisting aneurysm should occur before the pituitary tumor resection if there is a close location relationship. Nevertheless, for complicated pituitary apoplexy with significant tumor mass symptoms, such as crucial visual acuity or field defects with disturbed consciousness, ${ }^{3,12}$ treatment with decompression may be urgent and could be performed with interventional treatment of an aneurysm.

In this patient, the aneurysm was not demonstrated on preoperative images because of the complete occlusion of the left ICA. The aneurysm was visualized after recanalization of the artery by tumor decompression. Thus, tumor excision was first performed in this patient without caution regarding the coexisting aneurysm, which, in our opinion, may be dangerous if accidental rupture of the aneurysm with intractable bleeding occurs during resection because of a 



FIG. 3. Preoperative gadolinium-enhanced coronal (A) and sagittal (B) MRI on day 2 after admission showing a large pituitary tumor involving a bilateral cavernous segment of the ICA and causing occlusion of the left ICA. Postoperative gadolinium-enhanced coronal (C) and sagittal (D) MRI 3 months after operation demonstrating a gross-total resection of the tumor and recanalization of ICA on the left side (C, white arrow) and high-signal fat tissue in pituitary fossa. Intraoperative images $(E-G)$ indicating the tumor invading the bottom of saddle $(\mathbf{E})$, bleeding tumor tissue $(\mathbf{F})$, and close reconstruction of skull base using fat tissue $(\mathbf{G})$.

mistake in manipulation or hemodynamic change in arterial reperfusion. Fortunately, this patient experienced safe surgery without any bleeding accidents or enlargement of cerebral infarction, which may be attributed to the meticulous procedures, effective control of blood pressure and intracranial pressure, and the already compensating blood flow contralateral to blood circulation.

In our patient, occlusion of the left ICA was due to compression by pituitary apoplexy. After removing the hemorrhagic pituitary adenoma, no additional cerebral infarction was found, which may be due to the compensatory effect formed by the opening of the collateral circulation. Derdeyn et al. reported that when severe stenosis or occlusion occurs on one side of the carotid artery, compensation of the circle of Willis plays a vital role. ${ }^{17}$ In contrast, the ACOA and PCOA involve essential collateral circulation of the circle of Willis. In previous reports, the brain was less likely to be ischemic after carotid occlusion in individuals with collateral circulation of the circle of Willis. The more adequate the collateral blood flow, the less neurological impairment, ${ }^{18}$ and the effect of ACOA was particularly pronounced. In this case, the patient experienced early cerebral ischemia due to acute occlusion of ICA, which resulted in worsening of symptoms and was followed by no further worsening of cerebral infarction due to the formation of collateral circulation by the timely removal of the tumor. This is supported by marked thickening of the ACOA on the right side.
Presently, no cases of pituitary apoplexy-related ICA occlusion accompanied by a concealed aneurysm have been reported, ${ }^{4,5}$ and surgery for simple decompression may be hazardous, although our patient might be seen as the lucky one. Thus, radiological evaluation before resection is critical to screen for possible coexisting aneurysm, especially in patients with occlusive ICA, to avoid unexpected hemorrhage. Furthermore, we suggest that patients with large pituitary adenomas or pituitary apoplexy (notably involving the ICA or cavernous sinus) who have precipitating factors for formation of an aneurysm (e.g., history of primary hypertension or diabetes, family history of aneurysms, and older age $)^{4,5,19,20}$ should receive preoperative radiological evaluation with CTA or digital subtraction angiography (DSA), except for routinely enhanced MRI. Notably, for patients with precipitating factors for intracranial aneurysm who show no development of aneurysm on radiological evaluation, careful manipulation with better control of blood pressure during surgery is critical.

\section{Lessons}

Occlusion of the ICA with no development of an aneurysm due to compression by an apoplectic pituitary adenoma is rare. For patients with pituitary apoplexy accompanied by a rapid decrease in neurological conditions, emergency decompression surgery with EETS resection can achieve satisfactory results. However, with occlusion of ICA by enlarged pituitary adenoma or pituitary apoplexy, a hidden but 
rare intracranial aneurysm may be considered, and intraoperative caution is essential. Performing CTA or DSA before and after surgery can effectively reduce the missed diagnosis or misdiagnosis of this comorbidity and avoid life-threatening bleeding events from accidental rupture of the aneurysm.

\section{Limitation of the Study}

We acknowledge the limitation in this study. Our observation is somewhat hypothetical because we did not perform DSA based only on the CTA. Therefore, it is not possible to generalize our experience. Nevertheless, this case may contribute to future treatment options for pituitary apoplexy because of the specificity of this patient's comorbidities.

\section{References}

1. Zhang C, Feng F, Zhu Y, Wang R, Xing B. Cerebral infarction caused by pituitary apoplexy: case report and review of literature. Turk Neurosurg. 2014;24(5):782-787.

2. Rey-Dios R, Payner TD, Cohen-Gadol AA. Pituitary macroadenoma causing symptomatic internal carotid artery compression: surgical treatment through transsphenoidal tumor resection. J Clin Neurosci. 2014;21(4):541-546.

3. Briet C, Salenave S, Bonneville JF, Laws ER, Chanson P. Pituitary apoplexy. Endocr Rev. 2015;36(6):622-645.

4. Hu J, Lin Z, Zhang Y, et al. Prevalence of unruptured intracranial aneurysms coexisting with pituitary adenomas. World Neurosurg. 2019;126:e526-e533.

5. Oh MC, Kim EH, Kim SH. Coexistence of intracranial aneurysm in 800 patients with surgically confirmed pituitary adenoma. J Neurosurg. 2012;116(5):942-947.

6. Pant B, Arita K, Kurisu K, Tominaga A, Eguchi K, Uozumi T. Incidence of intracranial aneurysm associated with pituitary adenoma. Neurosurg Rev. 1997;20(1):13-17.

7. Sasagawa Y, Tachibana O, Shiraga S, Takata H, Akai T, lizuka H. A clinical feature and therapeutic strategy in pituitary adenomas associated with intracranial aneurysms. Article in Japanese. No Shinkei Geka. 2012;40(1):15-21.

8. Zhang RC, Mu YF, Dong J, Lin XQ, Geng DQ. Complex effects of apoplexy secondary to pituitary adenoma. Rev Neurosci. 2017;28(1):59-64.

9. Cardoso ER, Peterson EW. Pituitary apoplexy: a review. Neurosurgery. 1984;14(3):363-373.

10. Randeva HS, Schoebel J, Byrne J, Esiri M, Adams CB, Wass JA. Classical pituitary apoplexy: clinical features, management and outcome. Clin Endocrinol (Oxf). 1999;51(2):181-188.
11. Glezer A, Bronstein MD. Pituitary apoplexy: pathophysiology, diagnosis and management. Arch Endocrinol Metab. 2015;59(3): 259-264.

12. Grzywotz A, Kleist B, Möller LC, et al. Pituitary apoplexy—a single center retrospective study from the neurosurgical perspective and review of the literature. Clin Neurol Neurosurg. 2017;163:39-45.

13. Banerjee C, Snelling B, Hanft S, Komotar RJ. Bilateral cerebral infarction in the setting of pituitary apoplexy: a case presentation and literature review. Pituitary. 2015;18(3):352-358.

14. Dogan S, Kocaeli H, Abas F, Korfali E. Pituitary apoplexy as a cause of internal carotid artery occlusion. $J$ Clin Neurosci. 2008;15(4):480-483.

15. Peng Z, Tian D, Wang $H$, et al. Epistaxis and pituitary apoplexy due to ruptured internal carotid artery aneurysm embedded within pituitary adenoma. Int J Clin Exp Pathol. 2015;8(11):14189-14197.

16. Clark JD, Freer CE, Wheatley T. Pituitary apoplexy: an unusual cause of stroke. Clin Radiol. 1987;38(1):75-77.

17. Derdeyn CP, Khosla A, Videen TO, et al. Severe hemodynamic impairment and border zone-region infarction. Radiology. 2001;220(1):195-201.

18. Kluytmans M, van der Grond J, van Everdingen KJ, Klijn CJ, Kappelle LJ, Viergever MA. Cerebral hemodynamics in relation to patterns of collateral flow. Stroke. 1999;30(7):1432-1439.

19. Boulouis G, Rodriguez-Régent C, Rasolonjatovo EC, et al. Unruptured intracranial aneurysms: an updated review of current concepts for risk factors, detection and management. Rev Neurol (Paris). 2017;173(9):542-551.

20. Brown RD Jr, Broderick JP. Unruptured intracranial aneurysms: epidemiology, natural history, management options, and familial screening. Lancet Neurol. 2014;13(4):393-404.

\section{Disclosures}

The authors report no conflict of interest concerning the materials or methods used in this study or the findings specified in this paper.

\section{Author Contributions}

Conception and design: Zhu. Acquisition of data: Zhu, Xu. Analysis and interpretation of data: Zhu. Drafting the article: Zhu. Critically revising the article: Xiao, S. Xie, M.X. Xie. Reviewed submitted version of manuscript: Zhu.

\section{Correspondence}

Shun-Wu Xiao: Affiliated Hospital of Zunyi Medical University, Guizhou, China.xswlove1976@126.com. 